\title{
Recent Results from the VLBA
}

\section{P. J. DIAMOND AND A.J.BEASLEY}

National Radio Astronomy Observatory, ' Socorro, New Mexico, 87801

January 5, 1993

\section{Introduction}

The VLBA construction phase is now over and in the spring of 1993 it is hoped that full VLBA observing can begin. However the VLBA antennas have been operational for some time and several astronomy programs have been run on them. Below we shall present some of these latest results.

\section{Twisted pc-scale structure in the BL Lacertae object Mrk 501}

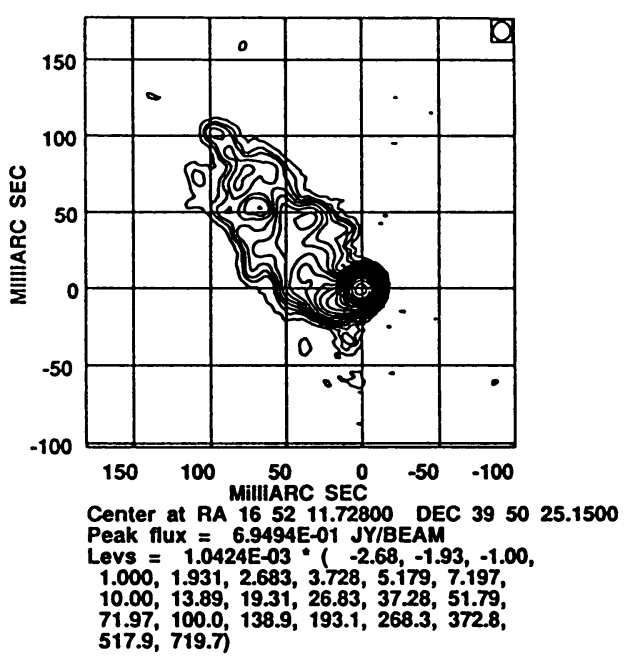

Fig. 1.- Mrk 501, VLBA MkII data

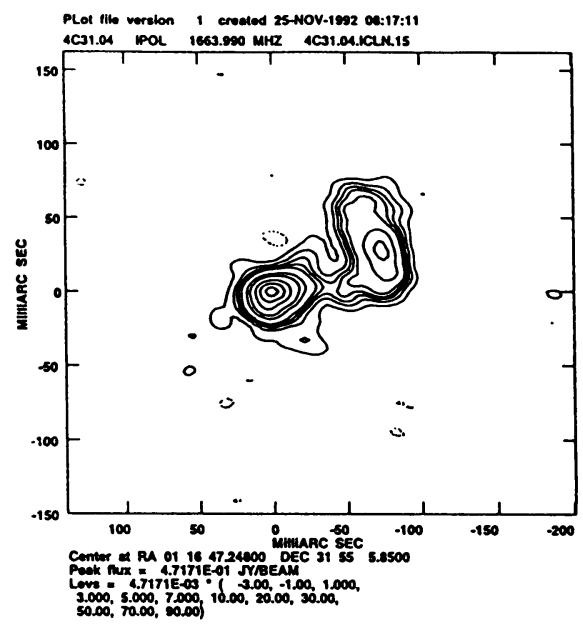

Fig. 2.-4C31.04, VLBA MkII data

The BL Lacertae object Mrk 501 is hosted by the $m_{p g}=13.7$ mag elliptical galaxy UGC 10599. The radio continuum structure of Mrk 501, shown in Fig.1, is very puzzling. It exhibits a one-sided "core-jet" whose elongation position angle appears to twist through about $79^{\circ}$ between 2 and 100 milliarcsecond (mas), or 1 and 46 $\mathrm{h}^{-1} \mathrm{pc}$, as revealed in published global VLBI, EVN, and VLA images. The origin of this dramatic structural twisting on pc scales is difficult to investigate with existing

- Associated Universities Inc. operates the National Radio Astronomy Observatory under National Science Foundation Cooperative Agreement No. AST-8814515 




Fig. 3.- HR1099, VLBA MkIII image

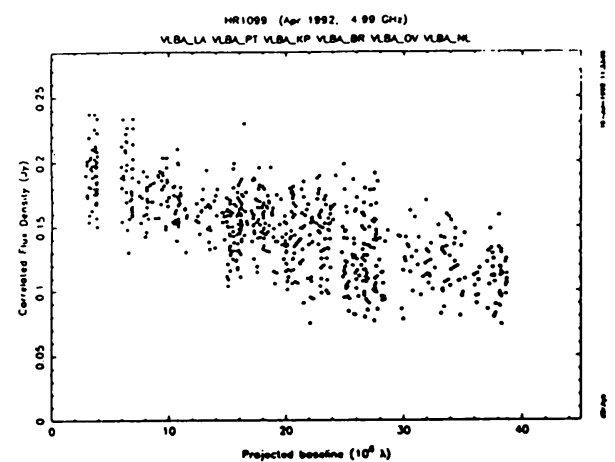

Fig. 4.- HR1099, Visibility function

radio data, since no image adequately follows the twist on scales of tens of mas. To remedy this problem , $6 \mathrm{~cm}$ Mark II continuum data on Mrk 501 were acquired during 1991 December with 4 antennas of the partially constructed NRAO VLBA, augmented by one VLA antenna. A naturally weighted image from this data set, obtained after only 3 interations of phase self-calibration and no amplitude selfcalibration, is shown in the figure. This image has an angular resolution of about 12 mas FWHM and a dynamic range (ratio of peak to off-source rms) of about 2400 . This naturally weighted image and a higher-resolution uniformly weighted one are being used to follow the details of the structural twist, and to interpret both the twist and other properties of Mrk 501 within the context of three possible models for the pronounced observed curvature. These observations were made by J. M. Wrobel and J. E. Conway.

\section{A VLBA Image of the radio galaxy $4 \mathrm{C31.04}$}

Figure 2 shows an image of the radio source $4 \mathrm{C} 31.04$ at $18 \mathrm{~cm}$ wavelength. This image was made using 7 VLBA antennas plus a single VLA antenna by Giovanninni et al. using the MkII recording system. 4C31.04 is identified with a nearby bright elliptical galaxy $(\mathrm{z}=0.06)$. The restoring beam size is $17 \times 10$ mas at $\mathrm{PA}=72 \mathrm{deg}$. The dynamic range of this image is in excess of $300: 1$. This source was not detected on transatlantic baselines and thus does not contain a signifigant high brightness core at this wavelength. If this object contains a jet it must have an apparent bend of approximately 90 degrees. These data were taken by Giovanninni et al. 


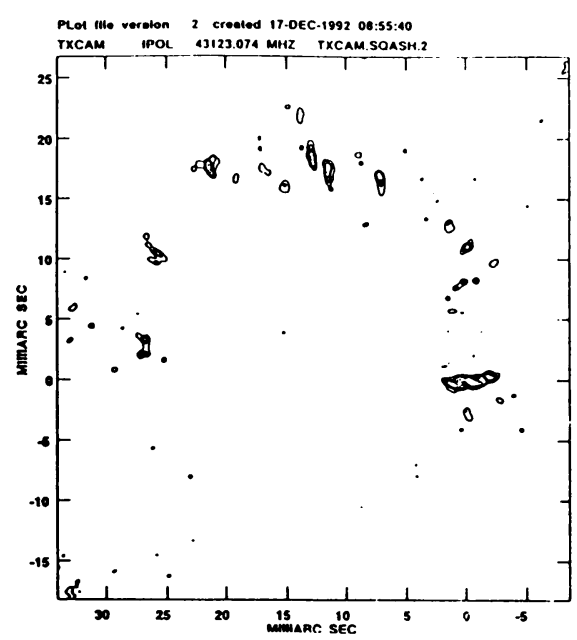

Fig. 5.- TXCAM: $\mathrm{SiO}$ masers

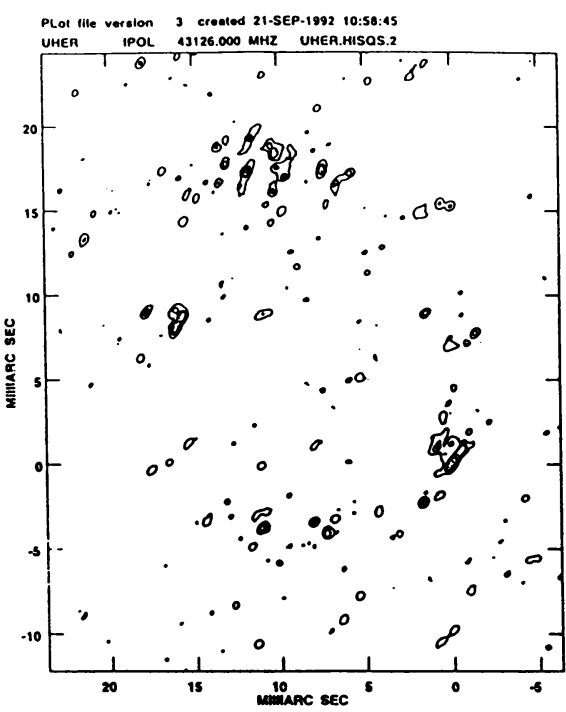

Fig. 6.- U Her: $\mathrm{SiO}$ masers

\section{MkIII VLBA Observations of the RS CVn system HR1099}

In Fig. 3 a $4.8 \mathrm{GHz}$ MkIII image of the active RS Cvn system HR1099 is presented. Observations of the binary star at X-ray, UV and radio wavelengths examine different aspects of the high levels of magnetic activity seen from such objects; at radio wavelengths we probe the coronal structures of the system. Intriguingly, high-resolution VLBA observations suggest an extended emission region surrounding the two stars, as indicated by the declining visibility-uv radius plot seen in Fig. 4. The derived angular size $(2.1 \mathrm{mas})$ is 1.5 times the overall size of the system, and suggests a possible "binary synergism" to the nonthermal radio emission seen from this system. Further observations and gyrosynchrotron modelling are currently underway. Data taken by Beasley et al.

\section{5. $43 \mathrm{GHz}$ VLBA Observations of SiO Masers around Late-type stars}

In Figs 5 and 6 we show VLBA images of the $\mathrm{SiO}$ masers around two late-type stars, U Herculis and TX Cam. These images were made from 4 (U Her) and 5 (TX Cam) station VLBA MkII data. Strong fringes were detected on most baselines demonstrating that the $\mathrm{SiO}$ masers are more compact than previously thought. The images of these two sources are the integrated emission over the whole maser velocity range and it is striking that in both cases we see clear evidence of a ring-like structure. It is probable that the masers lie in the gas just ejected by the star, or in the outer atmosphere of the star itself. More detailed analysis indicates that the masers may be situated in the gas that is decelerating after being ejected from the star and before the dust condensation point has been reached. Observations were performed by P.J.Diamond, J.Benson, V.Dhawan, W.Junor and A.Zensus. 